\section{IJ§ER}

ISSN: 2149-5939
International Journal of Social Sciences and Education Research

Online, http://dergipark.gov.tr/ijsser

Volume: 2(3), 2016

\title{
An EOQ model with defective items and shortages in fuzzy sets envi- ronment
}

\author{
Harun Sulak $^{1}$
}

Received Date: 17 / $01 / 2016$

Accepted Date: 22 / 04 / 2016

\begin{abstract}
The basic assumption of the classical inventory (EOQ/EPQ) models is that \%100 of items ordered/produced are perfect. However, in most of the production processes, it is unavoidable that the items ordered or produced are defective. This study develops a fuzzy economic order quantity model with defective items and shortages. In this model, the demand, defective rate, ordering cost, unit cost, disposal cost, holding cost, backordering cost and screening cost are expressed as trapezoidal and triangular fuzzy numbers. Moreover, the Graded Mean Integration Representation method is applied to defuzzify the total profit function in fuzzy sense, and then the optimal policy for the model is determined. The results between crisp case and fuzzy case are compared with the help of numerical examples. Finally, sensitivity analysis is carried out to find the effect of changes in the values of selling prices of good-and imperfect quality items, screening rate and percentage of scrap items on optimal solutions.
\end{abstract}

Keywords: Economic Order Quantity, Defective Items, Backorder, Graded Mean Integration Representation Method, Trapezoidal/Triangular Fuzzy Numbers

\section{Introduction}

The basic assumption of the classical Economic Order/Production Quantity (EOQ/EPQ) models is that $\% 100$ of ordered/produced items are perfect quality. By loosening this assumption and introducing some hypothesis, many new models related with different EOQ and EPQ models with percentage defective items have been developed by researchers.

Rosenblatt and Lee (1986) and Porteus (1986) considered an EPQ model without backorder where the production system contains some defective items. The basic assumption of their model is that production system produces only good-quality items from the starting point of production processes until a time point. In this time point which is a random variable, system becomes outof-control and starts to produce defective items with a percentage of production until end of the production time. This model was extended by Kim and Hong (1999) with the case of the distrubition of the time passes until system becomes out-of-control is arbitrarily distributed. Chung and Hou (2003) reconsidered an inventory model with backorder in which the aforementioned models were combined. Moreover, Hayek and Salameh (2001) proposed an EPQ model with defective items and shortages that percentage defective has uniform distribution. All the defective items were reworked in their model. Chiu (2003) extended the Hayek and Salameh model (2001) to the case that a portion of the defective items were reworked for making them good-quality item and the others were sold at a discounted price. Chan et al. (2003) proposed three EPQ models in which produced products classified as good quality, good quality after reworking, imperfect quality and

\footnotetext{
${ }^{1}$ Asst. Prof., Süleyman Demirel University, Faculty of Economics and Administrative Sciences, Department of Econ-
} ometrics, Isparta/TURKEY, harunsulak@sdu.edu.tr 
Sulak, H. (2016). An EOQ model with defective items and shortages in fuzzy sets environment. International Journal of Social Sciences and Education Research, 2 (3), 915-929.

scrap. The basic assumptions of these models were not allowing backorders and reworking time was zero. Eroglu et al. (2008) extended this model to the case that the shortages were allowed.

Besides these models, Salameh and Jaber (2000) proposed an EOQ model, where each lot contains percentage defectives which has a uniform distribution, and they assumed that shortages were not allowed. Goyal and Cardenas-Barron (2002) reworked on the paper and to find out the optimal lot size, they gave a practical approach. Papachristos and Konstantaras (2006) extended the Chen et al. (2003) and Salameh and Jaber (2000) models. They clarified the sufficient conditions for shortages given in these papers. In addition, Eroglu and Ozdemir (2007) rederived the Salameh and Jaber (2000) model to the case that shortages were allowed. In that model, they assumed that defective items contain imperfect quality and scrap. Scrap items were subtracted from inventory and imperfect quality items were sold as a single lot once screening process finished. Sulak et al. (2015) developped the Eroglu and Ozdemir (2007) model in case of permissible delay in payment. Khan et al. (2011) summarized the extensions of the EOQ model for imperfect quality items. Jaber et al. (2013) extended the model Salameh and Jaber (2000) model by assuming that the imperfect quality items were repaired or replaced by good ones.

The aforementioned models assume that the model parameters are described as a crisp values or having crisp statistical distributions to reflect uncertainty. Because of resolving the uncertainty which has a significant importance for the decision makers, several researchers have proposed different inventory problems in fuzzy sets environment.

Fuzzy set theory, introduced by Zadeh (1965), has been widely used among the researcher to treat classical inventory models. Park (1987) considered the classical EOQ model in fuzzy sense, where ordering cost and holding cost were trapezoidal fuzzy numbers. Chen et al. (1996) fuzzified the holding cost, ordering cost and backorder cost with trapezoidal fuzzy numbers. Yao and Chiang (2003) fuzzified an inventory model without backorder in which the demand and the holding cost were triangular fuzzy numbers. They used the signed distance and the centroid methods to defuzzify the total cost. Chang (2004) first developed an EOQ model with imperfect quality in fuzzy sense. In that paper, the annual demand and defective rate considered as triangular fuzzy numbers and used Signed Distance Method to obtain the optimal order quantity. Vijayan and Kumaran (2008) considered inventory models with partial backorders and lost sales an fuzzy stock-out periods. Halim et al. (2009) considered an EPQ model in which rate of the defective items and parameter $\gamma$ in the probability density function were tringular fuzzy numbers. And they used the centroid method to defuzzify cost per unit time in fuzzy sense. Björk (2009) proposed a fuzzy EOQ model in which the demand and lead time were triangular fuzzy numbers. $\mathrm{Hu}$ et al. (2010) extended the model eroğlu and Ozdemir (2007) model by introducing service level constrains. They fuzzified the demand and defective rate as trapezoidal fuzzy numbers and used the signed distance method to defuzzify the total profit per unit time in fuzzy sense. Jaggi et al. (2012) developed a fuzzy economic order quantity model for deteriorating items with shortages. They fuzzified the demand, holding cost, unit variable cost, shortage cost and deterioration rate into triangular fuzzy numbers. The total cost function defuzzified by using the Graded Mean Integration Representation, Signed Distance and Centroid Methods. Kumar and Goswami (2013) fuzzified an EOQ model for imperfect quality items and shortages. The annual demand and defective items rate were considered as triangular fuzzy numbers. To obtain total profit per cycle in the fuzzy sense, fuzzy random renewal reward theorem was applied. Chang et al. (1998) discussed 
Sulak, H. (2016). An EOQ model with defective items and shortages in fuzzy sets environment. International Journal of Social Sciences and Education Research, 2 (3), 915-929.

the classic inventory model with backorder in fuzzy sense, where backorder quantity were a triangular fuzzy number. Lin and Yao (2000) developed an inventory problem in fuzzy sense in which the production quantity was a trapezoidal fuzzy number. They used the Extension Principle and Centroid Method for obtaining the optimal production quantity in a cycle. Hesieh (2002) proposed two fuzzy production inventory models. The annual demand, inventory cost, set up cost, production rate and the production quantity were considered in fuzzy sense in the first model while the production quantity and the parameters were defined as trapezoidal fuzzy numbers in the second model. Then, the Function Principle, Graded Mean Integration Representation Method and Extension of Lagrange Method were used to get fuzzy total production inventory cost and the optimal production quantity in fuzzy sense. Chen et al. (2007) fuzzified an EPQ model with imperfect quality items sold at a discounted price. They considered that the daily holding cost, setup cost, the daily demand and the production quantity were trapezoidal fuzzy numbers. Björk (2008) was developed an EPQ model without shortages under the fuzzy decision variable and cycle time.

Kazemi et al. (2010) considered an inventory model with backorders in fuzzy sense. In that model, all parameters, the optimal order size and optimal backorder quantity were fuzzified by using trapezoidal and triangular fuzzy numbers. Mahata and Goswami (2013) developed an EOQ model for items with imperfect quality and shortage backordering in fuzzy environments. They assumed that the model parameters and the optimal order size and optimal backorder quantity were fuzzified by employing two types of fuzzy numbers, which are trapezoidal and triangular. Similar problems to that of Björk (2008) and to the one in the paper are presented in Chen et al. (2005), Chen and Chang (2008) and Vijayan and Kumaran (2009).

In this paper, we propose a fuzzy economic order quantity model with defective items and shortages based on the work of Eroglu and Ozdemir (2007). In this model, the demand, defective rate, ordering cost, unit cost, disposal cost, holding cost, backordering cost and screening cost are considered as trapezoidal and triangular fuzzy numbers. Moreover, the Graded Mean Integration Representation method is applied to defuzzify the total profit function in fuzzy sense, and then the optimal policy for the model is determined. The results between the crisp case and fuzzy case are compared with the help of numerical examples.

\section{The Crisp model}

Eroglu and Ozdemir (2007) developed an EOQ model with defective items and shortages. They considered the following situations:

- A lot size of $y$ is replenished instantaneously with a unit purshasing price of $c$ and ordering cost $k$.

- Each lot contains percentage defectives $p$. Each lot received is screened $100 \%$ with a screening rate per unit time of $x$.

- Defective items contain imperfect quality items and scrap items with a rate of $1-\theta$ and $\theta$, respectively.

- When the screening process finishes, imperfect quality items are sold as a single lot and scrap items are subtracted from inventory with unit $\operatorname{cost}$ of $c_{s}$. The selling price of good and imperfect-quality items are $s$ and $v$ per unit, respectively, where $s>v$.

- To eliminate backorders the following constrains should be provided: $1-p-D / x>0$ and $x>D$. 
Sulak, H. (2016). An EOQ model with defective items and shortages in fuzzy sets environment. International Journal of Social Sciences and Education Research, 2 (3), 915-929.

- Screening time, $t_{3}$, must be at least equal or greater than the the expected value of the time $\left(t_{2}\right)$ to eliminate backorder.

The behaviour of the inventory level is illustrated in Fig. 1, where $t$ is the cycle lenght, $p y$ is the number of defective items subtracted from inventory and $t_{3}$ is the total screening time of the $y$ units ordered per cycle.

For a given percentage of defective items in each lot of size $y$ and shortages (the maximum backordering quantity is $w)$, the total profit per cycle $T P(y, w)$ is given as

$T P(y, w)=($ sales of good-quality items + sales of imperfect quality items $)$ (procurement cost + screening cost + disposal cost + shortage cost + holding cost)

$$
\begin{aligned}
=(s(1- & p) y+v(1-\theta) p y) \\
& -\left\{\left(c+d+c_{s} \theta p\right) y+k+\frac{h}{2}\left(\frac{2-D / x}{x}+\frac{(1-p-D / x)^{2}}{D}\right) y^{2}\right. \\
& \left.-\frac{h(1-p) w y}{D}+\frac{(h+\pi)(1-p) w^{2}}{2 D(1-p-D / x)}\right\}
\end{aligned}
$$

Moreover, dividing the total profit per cycle $T P(y, w)$ by the cycle lenght $t, t=(1-p) y / D$, the total profit per unit time (we denote it by $\mathcal{L}(y, w)$ ) of the model is given as follows:

$$
\begin{aligned}
\mathcal{L}(y, w)= & D\left(s-v(1-\theta)+c_{s} \theta+\frac{h y}{x}\right)+D\left(v(1-\theta)-c_{s} \theta-c-d-\frac{k}{y}-\frac{h y}{x}\right)\left(\frac{1}{1-p}\right) \\
& -\frac{h y}{2}(1-p)+h w-\frac{(h+\pi) w^{2}}{2(1-p-D / x) y}
\end{aligned}
$$

Besides, Eroglu and Ozdemir (2007) considered the uncertainty of defective rate and assumed that percentage of defective items $p$ is a random variable with a known probability density function $f(p)$, and then they derived the optimal order size and backorder quantity by maximizing the expected value of $\mathcal{L}(y, w)$.

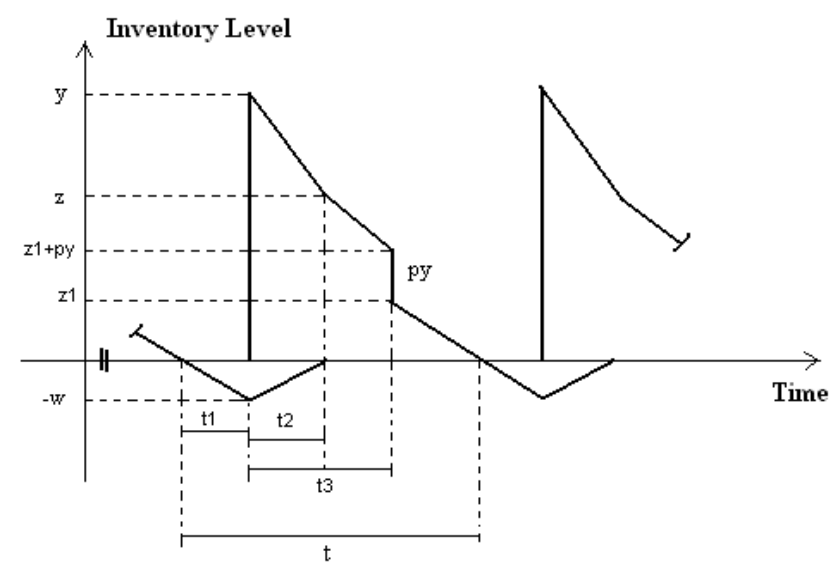

Fig. 1. Behaviour of the inventory level over time.

As we know, Formula (2) is the crisp model. Because $\mathcal{L}(y, w)$ is strictly concave (see Appendix A), partial derivatives of $\mathcal{L}(y, w)$ with respect to $y$ and $w$ are set equal to zero separately to get optimum values of $y^{*}$ and $w^{*}$.

Then, $y^{*}$ and $w^{*}$ are given as follows: 
Sulak, H. (2016). An EOQ model with defective items and shortages in fuzzy sets environment. International Journal of Social Sciences and Education Research, 2 (3), 915-929.

$$
\begin{aligned}
& y_{c}^{*}=\sqrt{\frac{2 k D}{h\left((1-p)^{2}+\frac{2 p D}{x}-\frac{h(1-p)(1-p-D / x)}{(h+\pi)}\right)}} \\
& w_{c}^{*}=\frac{h(1-p-D / x)}{(h+\pi)} y_{c}^{*}
\end{aligned}
$$

\section{Solution methodology and graded mean integration representation method}

Before presenting the fuzzy inventory model, we give some basic definitions and properties, taken from Chen (1985) and Mizumoto and Tanaka (1979), about fuzzy numbers and fuzzy sets with relevant operations.

Definition 1. A fuzzy number $\tilde{B}$ in the real line $R$ is a fuzzy set characterized by a membership function $\mu_{\tilde{B}}$ as

$$
\mu_{\tilde{B}}: R \rightarrow[0,1]
$$

Definition 2. A fuzzy number $\tilde{B}$ is defined as

$$
\tilde{B}=\int \frac{\mu_{\widetilde{B}}(x)}{x}
$$

with the understanding that $\mu_{\tilde{B}}(x) \in[0,1]$ represents the grade of membership of $x$ in $\tilde{B}$.

Definition 3. A fuzzy number $\widetilde{B}$ in $R$ is said to be fuzzy convex if for any real number $x, y, z \in$ $R$ with $x \leq y \leq z$,

$$
\mu_{\tilde{B}}(y) \geq \mu_{\tilde{B}}(x) \wedge \mu_{B}(z)
$$

where $\wedge$ denotes the minimum operator.

Definition 4. A fuzzy set $\tilde{B}$ on $R$ is said to be normal if there exists an $x_{0} \in R$, such that $\mu_{\tilde{B}}\left(x_{0}\right)=1$.

Definition 5. A fuzzy set $\tilde{Z}=(a, b, c, d)$ on $R$, where $a<b<c<d$, is called a trapezoidal fuzzy number if its membership function is

$$
\mu_{\tilde{Z}}(x)= \begin{cases}p(x)=\frac{x-a}{b-a} & a \leq x \leq b, \\ 1 & b \leq x \leq c, \\ r(x)=\frac{d-x}{d-c} & c \leq x \leq d, \\ 0, & \text { otherwise. }\end{cases}
$$

When $b=c$, the trapezoidal fuzzy number becomes a triangular fuzzy number.

In this paper, we recall some arithmetical operations under the function principle as follows:

Assume that $\widetilde{N}=\left(n_{1}, n_{2}, n_{3}, n_{4}\right)$ and $\tilde{L}=\left(l_{1}, l_{2}, l_{3}, l_{4}\right)$ are two trapezoidal fuzzy numbers. Then, we have

- $\tilde{N} \oplus \tilde{L}=\left(n_{1}+l_{1}, n_{2}+l_{2}, n_{3}+l_{3}, n_{4}+l_{4}\right)$.

- If $n_{i}, l_{i}>0$, for $i=1,2,3,4$, then we have

$\widetilde{N} \otimes \tilde{L}=\left(n_{1} l_{1}, n_{2} l_{2}, n_{3} l_{3}, n_{4} l_{4}\right)$.

- $\quad$ Since $\ominus \tilde{L}=\left(-l_{4},-l_{3},-l_{2},-l_{1}\right)$, the subtraction of $\widetilde{N}$ and $\tilde{L}$ is

$$
\tilde{N} \ominus \tilde{L}=\tilde{N} \oplus(\ominus \tilde{L})=\left(n_{1}-l_{4}, n_{2}-l_{3}, n_{3}-l_{2}, n_{4}-l_{1}\right) \text {. }
$$


Sulak, H. (2016). An EOQ model with defective items and shortages in fuzzy sets environment. International Journal of Social Sciences and Education Research, 2 (3), 915-929.

- $\quad 1 \oslash \tilde{L}=\tilde{L}^{-1}=\left(\frac{1}{l_{4}}, \frac{1}{l_{3}}, \frac{1}{l_{2}}, \frac{1}{l_{1}}\right), \quad$ where $n_{i}>0$ for each $i=1,2,3,4$.

$$
\text { If } n_{i}, l_{i}>0 \text { for } i=1,2,3,4 \text {, then we get } \widetilde{N} \oslash \tilde{L}=\left(\frac{n_{1}}{l_{4}}, \frac{n_{2}}{l_{3}}, \frac{n_{3}}{l_{2}}, \frac{n_{4}}{l_{1}}\right) \text {. }
$$

Let $\ell \in \mathrm{R}$, then $\quad \ell \odot \widetilde{\mathrm{N}}=\ell \otimes \widetilde{\mathrm{N}}= \begin{cases}\left(\ell \mathrm{n}_{1}, \ell \mathrm{n}_{2}, \ell \mathrm{n}_{3}, \ell \mathrm{n}_{4}\right) & \ell \geq 0 \\ \left(\ell \mathrm{n}_{4}, \ell \mathrm{n}_{3}, \ell \mathrm{n}_{2}, \ell \mathrm{n}_{1}\right) & \ell<0 .\end{cases}$

Now, we define the graded mean integration representation (GMIR), a defuzzification method introduced by Chen and Hesieh (1998), to defuzzify the fuzzy total profit per unit time function developed in later section. In the graded mean integration representation method, the functions $p^{-1}$ and $r^{-1}$ are the inverse functions of $p$ and $r$, respectively. We also describe the graded mean $\alpha-$ level value of $\widetilde{N}$ as

$$
\frac{\alpha\left(\left(p^{-1}(\alpha)+r^{-1}(\alpha)\right)\right.}{2}
$$

Let $\widetilde{N}=\left(n_{1}, n_{2}, n_{3}, n_{4}\right)$ be a trapezoidal fuzzy number, then the graded mean integration representation of $\widetilde{N}$ can be obtained as

$$
\begin{aligned}
D F(\widetilde{N}) & =\frac{\int_{0}^{1} \frac{\alpha\left(\left(p^{-1}(\alpha)+r^{-1}(\alpha)\right)\right.}{2} d \alpha}{\int_{0}^{1} \alpha d \alpha}=\int_{0}^{1} \alpha\left(\left(p^{-1}(\alpha)+r^{-1}(\alpha)\right) d \alpha\right. \\
& =\frac{n_{1}+2 n_{2}+2 n_{3}+n_{4}}{6},
\end{aligned}
$$

where $p^{-1}(\alpha)=n_{1}-\left(n_{1}-n_{2}\right) \alpha, r^{-1}(\alpha)=n_{4}+\left(n_{3}-n_{4}\right) \alpha$.

In particular if we take $n_{2}=n_{3}=n$, then we derived the triangular fuzzy number $\widetilde{N}_{1}=$ $\left(n_{1}, n, n_{4}\right)$. In this case, using the graded mean integration representation method, we calculate

$$
\operatorname{DF}\left(\widetilde{N}_{1}\right)=\frac{n_{1}+4 n+n_{4}}{6}
$$

\section{Fuzzy EOQ model with defective items and shortages}

In this section, we reconsider the crisp model given in equality (2) with combining the fuzziness of the demand, defective rate, ordering cost, unit cost, disposal cost, holding cost, backordering cost and screening cost $\left(D, q=1-p, K, c, c_{s}, h, \pi\right.$ and $\left.d\right)$. In this case, the total profit per unit time in fuzzy sense is given as

$$
\begin{aligned}
\tilde{\mathcal{L}}(y, w)= & \widetilde{D} \otimes\left(s \ominus v \oplus \theta \otimes\left(v \oplus \tilde{c}_{s}\right)\right) \oplus(\widetilde{D} \oslash \tilde{q}) \otimes\left(v \ominus \tilde{c} \ominus \tilde{d} \ominus \theta \otimes\left(v \oplus \tilde{c}_{s}\right)\right) \\
& \oplus(\widetilde{D} \otimes \tilde{h} \oslash x \ominus(\widetilde{D} \otimes \tilde{h}) \oslash(\tilde{q} \otimes x) \ominus \tilde{h} \otimes \tilde{q} \oslash 2) \otimes y \\
& \ominus\left(\widetilde{D} \otimes \widetilde{K} \oslash \tilde{q} \oplus((\tilde{h} \oplus \tilde{\pi}) \oslash[\tilde{q} \ominus(\widetilde{D} \oslash x)]) \otimes w^{2} \oslash 2\right) \oslash y \\
& \oplus \tilde{h} \otimes w
\end{aligned}
$$

where $\oplus, \ominus, \otimes$ and $\oslash$ are the fuzzy arithmetical operations under Function Principle. In this equation, we assume that $\widetilde{K}=\left(K_{1}, K_{2}, K_{3}, K_{4}\right), \widetilde{D}=\left(D_{1}, D_{2}, D_{3}, D_{4}\right), \tilde{c}=\left(c_{1}, c_{2}, c_{3}, c_{4}\right), \tilde{q}=$ $\left(q_{1}, q_{2}, q_{3}, q_{4}\right), \quad \tilde{c}_{s}=\left(c_{s_{1}}, c_{s_{2}}, c_{s_{3}}, c_{s_{4}}\right), \quad \tilde{h}=\left(h_{1}, h_{2}, h_{3}, h_{4}\right), \quad \tilde{\pi}=\left(\pi_{1}, \pi_{2}, \pi_{3}, \pi_{4}\right), \quad \tilde{d}=$ $\left(d_{1}, d_{2}, d_{3}, d_{4}\right)$ are non-negative trapezoidal fuzzy numbers and

$\widetilde{D} \otimes\left(s \ominus v \oplus \theta \otimes\left(v \oplus \tilde{c}_{s}\right)\right)$ 
Sulak, H. (2016). An EOQ model with defective items and shortages in fuzzy sets environment. International Journal of Social Sciences and Education Research, 2 (3), 915-929.

$$
\begin{aligned}
= & \left(D_{1}\left(s-v+\theta\left(v+c_{s_{1}}\right)\right), D_{2}\left(s-v+\theta\left(v+c_{s_{2}}\right)\right),\right. \\
& \left.D_{3}\left(s-v+\theta\left(v+c_{s_{3}}\right)\right), D_{4}\left(s-v+\theta\left(v+c_{s_{4}}\right)\right)\right),
\end{aligned}
$$

$(\widetilde{D} \oslash \tilde{q}) \otimes\left(v \ominus \tilde{c} \ominus \tilde{d} \ominus \theta \otimes\left(v \oplus \tilde{c}_{s}\right)\right)$

$$
\begin{aligned}
= & \left(\frac{D_{1}\left(v-c_{4}-d_{4}-\theta\left(v+c_{S_{4}}\right)\right)}{q_{4}}, \frac{D_{2}\left(v-c_{3}-d_{3}-\theta\left(v+c_{S_{3}}\right)\right)}{q_{3}}\right. \\
& \left.\frac{D_{3}\left(v-c_{2}-d_{2}-\theta\left(v+c_{S_{2}}\right)\right.}{q_{2}}, \frac{D_{4}\left(v-c_{1}-d_{1}-\theta\left(v+c_{S_{1}}\right)\right)}{q_{1}}\right),
\end{aligned}
$$

$(\widetilde{D} \otimes \tilde{h} \oslash x \ominus(\widetilde{D} \otimes \tilde{h}) \oslash(\tilde{q} \otimes x) \ominus \tilde{h} \otimes \tilde{q} \oslash 2) \otimes y$

$$
\begin{aligned}
= & \left(\left(\frac{D_{1} h_{1}}{x}-\frac{D_{4} h_{4}}{q_{1} x}-\frac{h_{4} q_{4}}{2}\right) y,\left(\frac{D_{2} h_{2}}{x}-\frac{D_{3} h_{3}}{q_{2} x}-\frac{h_{3} q_{3}}{2}\right) y,\right. \\
& \left.\left(\frac{D_{3} h_{3}}{x}-\frac{D_{2} h_{2}}{q_{3} x}-\frac{h_{2} q_{2}}{2}\right) y,\left(\frac{D_{4} h_{4}}{x}-\frac{D_{1} h_{1}}{q_{4} x}-\frac{h_{1} q_{1}}{2}\right) y\right),
\end{aligned}
$$

$\ominus\left(\widetilde{D} \otimes \widetilde{K} \oslash \tilde{q} \oplus((\tilde{h} \oplus \tilde{\pi}) \oslash[\tilde{q} \ominus(\widetilde{D} \oslash x)]) \otimes w^{2} \oslash 2\right) \oslash y$

$$
\begin{aligned}
=( & -\frac{D_{4} K_{4}}{q_{1} y}-\frac{\left(h_{4}+\pi_{4}\right) w^{2}}{2\left(q_{1}-\left(D_{4} / x\right)\right) y},-\frac{D_{3} K_{3}}{q_{2} y}-\frac{\left(h_{3}+\pi_{3}\right) w^{2}}{2\left(q_{2}-\left(D_{3} / x\right)\right) y^{\prime}} \\
& \left.-\frac{D_{2} K_{2}}{q_{3} y}-\frac{\left(h_{2}+\pi_{2}\right) w^{2}}{2\left(q_{3}-\left(D_{2} / x\right)\right) y},-\frac{D_{1} K_{1}}{q_{4} y}-\frac{\left(h_{1}+\pi_{1}\right) w^{2}}{2\left(q_{4}-\left(D_{1} / x\right)\right) y}\right),
\end{aligned}
$$

$\tilde{h} \otimes w=\left(h_{1} w, h_{2} w, h_{3} w, h_{4} w\right)$

Substituting equations (12)-(16) in equation (11), then the fuzzy total profit per unit time function is written as

$$
\tilde{\mathcal{L}}(y, w)=\left(\mathcal{L}_{1}, \mathcal{L}_{2}, \mathcal{L}_{3}, \mathcal{L}_{4}\right)
$$

where

$$
\begin{aligned}
\mathcal{L}_{1}= & D_{1}\left(s-v+\theta\left(v+c_{S_{1}}\right)\right)+\frac{D_{1}\left(v-c_{4}-d_{4}-\theta\left(v+c_{S_{4}}\right)\right)}{q_{4}} \\
& +\left(\frac{D_{1} h_{1}}{x}-\frac{D_{4} h_{4}}{q_{1} x}-\frac{h_{4} q_{4}}{2}\right) y-\frac{D_{4} K_{4}}{q_{1} y}-\frac{\left(h_{4}+\pi_{4}\right) w^{2}}{2\left(q_{1}-\left(\frac{D_{4}}{x}\right)\right) y}+h_{1} w, \\
\mathcal{L}_{2}= & D_{2}\left(s-v+\theta\left(v+c_{S_{2}}\right)\right)+\frac{D_{2}\left(v-c_{3}-d_{3}-\theta\left(v+c_{S_{3}}\right)\right)}{q_{3}} \\
& +\left(\frac{D_{2} h_{2}}{x}-\frac{D_{3} h_{3}}{q_{2} x}-\frac{h_{3} q_{3}}{2}\right) y-\frac{D_{3} K_{3}}{q_{2} y}-\frac{\left(h_{3}+\pi_{3}\right) w^{2}}{2\left(q_{2}-\left(\frac{D_{3}}{x}\right)\right) y}+h_{2} w, \\
\mathcal{L}_{3}= & D_{3}\left(s-v+\theta\left(v+c_{S_{3}}\right)\right)+\frac{D_{3}\left(v-c_{2}-d_{2}-\theta\left(v+c_{S_{2}}\right)\right)}{q_{2}} \\
& +\left(\frac{D_{3} h_{3}}{x}-\frac{D_{2} h_{2}}{q_{3} x}-\frac{h_{2} q_{2}}{2}\right) y-\frac{D_{2} K_{2}}{q_{3} y}-\frac{\left(h_{2}+\pi_{2}\right) w^{2}}{2\left(q_{3}-\left(\frac{D_{2}}{x}\right)\right) y}+h_{3} w, \\
\mathcal{L}_{4}= & D_{4}\left(s-v+\theta\left(v+c_{S_{4}}\right)\right)+\frac{D_{4}\left(v-c_{1}-d_{1}-\theta\left(v+c_{S_{1}}\right)\right)}{q_{1}} \\
& +\left(\frac{D_{4} h_{4}}{x}-\frac{D_{1} h_{1}}{q_{4} x}-\frac{h_{1} q_{1}}{2}\right) y-\frac{D_{1} K_{1}}{q_{4} y}-\frac{\left(h_{1}+\pi \pi_{1}\right) w^{2}}{2\left(q_{4}-\left(\frac{D_{1}}{x}\right)\right) y}+h_{4} w .
\end{aligned}
$$


Sulak, H. (2016). An EOQ model with defective items and shortages in fuzzy sets environment. International Journal of Social Sciences and Education Research, 2 (3), 915-929.

Now, we defuzzify the fuzzy number $\tilde{\mathcal{L}}(y, w)$ by using the GMIR method. We get

$$
\begin{aligned}
D F(\tilde{\mathcal{L}}(y, w))=\frac{1}{6}\{ & \left(D_{1}\left(s-v+\theta\left(v+c_{S_{1}}\right)\right)+\frac{D_{1}\left(v-c_{4}-d_{4}-\theta\left(v+c_{s_{4}}\right)\right)}{q_{4}}\right. \\
& \left.+\left(\frac{D_{1} h_{1}}{x}-\frac{D_{4} h_{4}}{q_{1} x}-\frac{h_{4} q_{4}}{2}\right) y-\frac{D_{4} K_{4}}{q_{1} y}-\frac{\left(h_{4}+\pi_{4}\right) w^{2}}{2\left(q_{1}-\left(\frac{D_{4}}{x}\right)\right) y}+h_{1} w\right) \\
+ & 2\left(D_{2}\left(s-v+\theta\left(v+c_{S_{2}}\right)\right)+\frac{D_{2}\left(v-c_{3}-d_{3}-\theta\left(v+c_{s_{3}}\right)\right)}{q_{3}}\right. \\
& \left.+\left(\frac{D_{2} h_{2}}{x}-\frac{D_{3} h_{3}}{q_{2} x}-\frac{h_{3} q_{3}}{2}\right) y-\frac{D_{3} K_{3}}{q_{2} y}-\frac{\left(h_{3}+\pi_{3}\right) w^{2}}{2\left(q_{2}-\left(\frac{D_{3}}{x}\right)\right) y}+h_{2} w\right) \\
+ & 2\left(D_{3}\left(s-v+\theta\left(v+c_{S_{3}}\right)\right)+\frac{D_{3}\left(v-c_{2}-d_{2}-\theta\left(v+c_{S_{2}}\right)\right)}{q_{2}}\right. \\
& \left.+\left(\frac{D_{3} h_{3}}{x}-\frac{D_{2} h_{2}}{q_{3} x}-\frac{h_{2} q_{2}}{2}\right) y-\frac{D_{2} K_{2}}{q_{3} y}-\frac{\left(h_{2}+\pi_{2}\right) w^{2}}{2\left(q_{3}-\left(\frac{D_{2}}{x}\right)\right) y}+h_{3} w\right) \\
+ & \left(D_{4}\left(s-v+\theta\left(v+c_{S_{4}}\right)\right)+\frac{D_{4}\left(v-c_{1}-d_{1}-\theta\left(v+c_{S_{1}}\right)\right)}{q_{1}}\right. \\
& \left.\left.+\left(\frac{D_{4} h_{4}}{x}-\frac{D_{1} h_{1}}{q_{4} x}-\frac{h_{1} q_{1}}{2}\right) y-\frac{D_{1} K_{1}}{q_{4} y}-\frac{\left(h_{1}+\pi_{1}\right) w^{2}}{2\left(q_{4}-\left(\frac{D_{1}}{x}\right)\right) y}+h_{4} w\right)\right\} .
\end{aligned}
$$

We consider $D F(\tilde{\mathcal{L}}(y, w))$ as the estimate of the total profit per unit time in fuzzy sense. Our objective is to maximize the total profit per unit time in fuzzy sense. Because $D F(\tilde{\mathcal{L}}(y, w))$ is concave (see the Appendix B), the optimal order size $y^{*}$ and the maximum backorder quantity $w^{*}$ are obtained by differentiating $D F(\tilde{\mathcal{L}}(y, w))$ with respect to $y$ and then with respect to $w$, and setting the partial derivatives equal to zero, i.e.

$$
\begin{gathered}
\frac{\partial D F(\tilde{\mathcal{L}}(y, w))}{\partial y}=\frac{1}{6}\left(\frac{D_{1} h_{1}}{x}-\frac{D_{1} h_{1}}{q_{4} x}+\frac{D_{4} h_{4}}{x}-\frac{D_{4} h_{4}}{q_{1} x}-\frac{h_{1} q_{1}}{2}-\frac{h_{4} q_{4}}{2}\right) \\
+\frac{2}{6}\left(\frac{D_{2} h_{2}}{x}-\frac{D_{2} h_{2}}{q_{3} x}+\frac{D_{3} h_{3}}{x}-\frac{D_{3} h_{3}}{q_{2} x}-\frac{h_{2} q_{2}}{2}-\frac{h_{3} q_{3}}{2}\right) \\
+\frac{1}{6}\left(\left(\frac{\left(h_{1}+\pi_{1}\right)}{2\left(q_{4}-\frac{D_{1}}{x}\right)}+\frac{2\left(h_{2}+\pi_{2}\right)}{2\left(q_{3}-\frac{D_{2}}{x}\right)}+\frac{2\left(h_{3}+\pi_{3}\right)}{2\left(q_{2}-\frac{D_{3}}{x}\right)}+\frac{\left(h_{4}+\pi_{4}\right)}{2\left(q_{1}-\frac{D_{4}}{x}\right)}\right) w^{2}\right. \\
\left.+\frac{D_{1} K_{1}}{q_{4}}+\frac{2 D_{2} K_{2}}{q_{3}}+\frac{2 D_{3} K_{3}}{q_{2}}+\frac{D_{4} K_{4}}{q_{1}}\right) \frac{1}{y^{2}}=0 \\
\text { and } \\
\frac{\partial D F(\tilde{\mathcal{L}}(y, w))}{\partial w}=\frac{1}{6}\left(h_{1}+2 h_{2}+2 h_{3}+h_{4}\right) \\
-\frac{1}{6}\left(\frac{\left(h_{1}+\pi_{1}\right)}{\left(q_{4}-\frac{D_{1}}{x}\right)}+\frac{2\left(h_{2}+\pi_{2}\right)}{\left(q_{3}-\frac{D_{2}}{x}\right)}+\frac{2\left(h_{3}+\pi_{3}\right)}{\left(q_{2}-\frac{D_{3}}{x}\right)}+\frac{\left(h_{4}+\pi_{4}\right)}{\left(q_{1}-\frac{D_{4}}{x}\right)}\right) \frac{w}{y}=0
\end{gathered}
$$

This yields to

$$
y^{*}=\sqrt{\frac{B}{-(F+2 M)-\frac{\left(h_{1}+2 h_{2}+2 h_{3}+h_{4}\right)^{2}}{2 H}}}
$$


Sulak, H. (2016). An EOQ model with defective items and shortages in fuzzy sets environment. International Journal of Social Sciences and Education Research, 2 (3), 915-929.

$w^{*}=\frac{\left(h_{1}+2 h_{2}+2 h_{3}+h_{4}\right)}{H} y^{*}$

where

$$
\begin{aligned}
& B=\frac{D_{1} K_{1}}{q_{4}}+\frac{2 D_{2} K_{2}}{q_{3}}+\frac{2 D_{3} K_{3}}{q_{2}}+\frac{D_{4} K_{4}}{q_{1}} \\
& F=\frac{D_{1} h_{1}}{x}-\frac{D_{1} h_{1}}{q_{4} x}+\frac{D_{4} h_{4}}{x}-\frac{D_{4} h_{4}}{q_{1} x}-\frac{h_{1} q_{1}}{2}-\frac{h_{4} q_{4}}{2} \\
& M=\frac{D_{2} h_{2}}{x}-\frac{D_{2} h_{2}}{q_{3} x}+\frac{D_{3} h_{3}}{x}-\frac{D_{3} h_{3}}{q_{2} x}-\frac{h_{2} q_{2}}{2}-\frac{h_{3} q_{3}}{2} \\
& H=\frac{\left(h_{1}+\pi_{1}\right)}{\left(q_{4}-\frac{D_{1}}{x}\right)}+\frac{2\left(h_{2}+\pi_{2}\right)}{\left(q_{3}-\frac{D_{2}}{x}\right)}+\frac{2\left(h_{3}+\pi_{3}\right)}{\left(q_{2}-\frac{D_{3}}{x}\right)}+\frac{\left(h_{4}+\pi_{4}\right)}{\left(q_{1}-\frac{D_{4}}{x}\right)} .
\end{aligned}
$$

The optimum total profit per unit time $D F(\tilde{\mathcal{L}}(y, w))$ is obtained by direct substitution of equations (21) and (22) into Equation (18).

When the demand, defective rate, and costs $\left(D, q=1-p, k, h, c, d, \pi\right.$ and $\left.c_{s}\right)$ are real numbers, that is

$k=k_{1}=k_{2}=k_{3}=k_{4}, h=h_{1}=h_{2}=h_{3}=h_{4}, c=c_{1}=c_{2}=c_{3}=c_{4}, d=d_{1}=d_{2}=$ $d_{3}=d_{4}, \pi=\pi_{1}=\pi_{2}=\pi_{3}=\pi_{4}, c_{s}=c_{s_{1}}=c_{s_{2}}=c_{s_{3}}=c_{S_{4}}, D=D_{1}=D_{2}=D_{3}=D_{4}, q=$ $q_{1}=q_{2}=q_{3}=q_{4}$, then the crisp model with defective items and shortages is presented. So, the following reduced forms of equations (21) and (22) are obtained:

$$
\begin{aligned}
& y_{c}^{*}=\sqrt{\frac{2 k D}{h\left((1-p)^{2}+\frac{2 p D}{x}+\frac{h(1-p)(1-p-D / x)}{(h+\pi)}\right)}} \\
& w_{c}^{*}=\frac{h(1-p-D / x)}{(h+\pi)} y_{c}^{*}
\end{aligned}
$$

If we assume that the demand, defective rate, and costs are triangular fuzzy numbers as

$$
\tilde{k}=\left(k_{1}, k, k_{2}\right), \widetilde{D}=\left(D_{1}, D, D_{2}\right), \tilde{c}=\left(c_{1}, c, c_{2}\right), \tilde{q}=\left(q_{1}, q, q_{2}\right), \tilde{c}_{s}=\left(c_{s_{1}}, c_{s}, c_{s_{2}}\right), \tilde{h}=
$$
$\left(h_{1}, h, h_{2}\right), \tilde{\pi}=\left(\pi_{1}, \pi, \pi_{2}\right), \tilde{d}=\left(d_{1}, d, d_{2}\right)$, then the equations (21) and (22) can be reduced the following equations:

$$
\begin{aligned}
& y^{*}=\sqrt{\frac{\frac{D_{1} K_{1}}{q_{2}}+\frac{4 K D}{q}+\frac{D_{2} K_{2}}{q_{1}}}{-\left(\frac{D_{1} h_{1}}{x}-\frac{D_{1} h_{1}}{q_{2} x}+\frac{D_{2} h_{2}}{x}-\frac{D_{2} h_{2}}{q_{1} x}-\frac{h_{1} q_{1}}{2}-\frac{h_{2} q_{2}}{2}+2\left(\frac{2 D h}{x}-\frac{2 D h}{q x}-\frac{2 h q}{2}\right)\right)-\frac{\left(h_{1}+4 h+h_{2}\right)^{2}}{2\left(\frac{\left(h_{1}+\pi \pi_{1}\right)}{\left(q_{2}-\frac{D_{1}}{x}\right)}+\frac{4(h+\pi)}{\left(q-\frac{D}{x}\right)}+\frac{\left(h_{2}+\pi_{2}\right)}{\left(q_{1}-\frac{D_{2}}{x}\right)}\right)}}} \\
& w^{*}=\left(\frac{\left(h_{1}+4 h+h_{2}\right)}{\left(\frac{\left(h_{1}+\pi_{1}\right)}{\left(q_{2}-\frac{D_{1}}{x}\right)}+\frac{4(h+\pi)}{\left(q-\frac{D}{x}\right)}+\frac{\left(h_{2}+\pi_{2}\right)}{\left(q_{1}-\frac{D_{2}}{x}\right)}\right)}\right) y^{*}
\end{aligned}
$$


Sulak, H. (2016). An EOQ model with defective items and shortages in fuzzy sets environment. International Journal of Social Sciences and Education Research, 2 (3), 915-929.

\section{Numerical examples}

To illustrate the behaviour of the fuzzy model, the following numerical examples are applied, and we compare the results between the fuzzy case and the crisp case.

Consider an inventory model with crisp parameters having the following values (from Eroglu and Ozdemir [12]: $D=15,000$ units/year, $k=\$ 400 /$ cycle, $h=\$ 4 /$ unit, $\pi=\$ 6 /$ unit, $x=$ $60,000 /$ units/year, $c=\$ 35 /$ unit, $d=\$ 1 /$ unit, $c_{s}=\$ 2 /$ unit, $s=\$ 60 /$ unit, $v=\$ 25 /$ unit, the portion of scrap items $\theta=\% 20$, but instead of assuming that the defective rate is uniformly distributed with mean 0.05 , we consider that the defective rate is around $p=0.05$ (i.e., the goodquality rate is $q=1-p=0.95$ ).

The optimal order size $y_{c}^{*}$, the optimal backorder quantity $w_{c}^{*}$, the optimum total profit per unit time $\mathcal{L}\left(y_{c}^{*}, w_{c}^{*}\right)$ of crisp model can be derived from equations (2)-(4), respectively. Then, we get $y_{c}^{*}=2129.59$ units, $w_{c}^{*}=596.28$ units and $G\left(y_{c}^{*}, w_{c}^{*}\right)=\$ 341,121.20$.

The optimal solution of the fuzzy model can be calculated in four cases by the GMIR method as follows:

Case 1. When the parameters $k, h, c, d, \pi, c_{s}, D$ and $q$ are trapezoidal fuzzy numbers as

$\tilde{k}=(300,350,450,500), \tilde{h}=(2,3.5,4.5,6), \tilde{c}=(25,30,40,45), \tilde{d}=(0.25,0.5,1.5,1.75)$, $\tilde{\pi}=(4,5.5,6.5,8), \widetilde{c_{S}}=(1,1.5,2.5,3), \widetilde{D}=(12000,14000,17000,18000)$,

$\tilde{q}=(0.93,0.94,0.96,0.99)$, then we have $D F(\tilde{\mathcal{L}}(y, w))=\$ 368,711.9, y^{*}=2149.85$ units and $w^{*}=585.72$ units.

Case 2. When the parameters $D$ and $q$ are trapezoidal fuzzy numbers defined as above, we have $D F(\tilde{\mathcal{L}}(y, w))=\$ 348,821.3, y^{*}=2143.07$ units and $w^{*}=594.53$ units.

Case 3. When the parameters $k, h, c, d, \pi, c_{S}, D$ and $q$ are triangular fuzzy numbers as

$$
\tilde{k}=(300,400,500), \tilde{h}=(2,4,6), \tilde{c}=(25,35,45), \tilde{d}=(0.25,1,1.75), \tilde{\pi}=(4,6,8),
$$

$\widetilde{c_{s}}=(1,2,3), \widetilde{D}=(12000,15000,18000), \tilde{q}=(0.93,0.95,0.99)$, then we have $D F(\tilde{\mathcal{L}}(y, w))=\$ 354,685.70, y^{*}=2125.60$ units and $w^{*}=586.64$ units.

Case 4. When the parameters $D$ and $q$ are triangular fuzzy numbers defined in the Case 3, then we have $D F(\tilde{\mathcal{L}}(y, w))=\$ 341,394.5, y^{*}=2124.42$ units and $w^{*}=595.12$ units.

Now, a sensitivity analysis is carried out to determine the effect of changes in the values of $s, v, x$ and $\theta$ on the optimal solution and the results are shown in the following Tables 1-4.

In Tables 1-4, we set some values of the parameters $(s, v, x, \theta)$. For each of these parameters, the variations in the values are arranged arbitrary. Besides, you can see the variations in the optimal values of the $y^{*}$ and $w^{*}$, and the total profit per unit time $D F(\tilde{\mathcal{L}}(y, w))$ between the second and fourth columns of these tables.

From Table 1, we can notice that when the unit selling price of good-quality items $s$ increases, the fuzzy total profit $D F(\tilde{\mathcal{L}}(y, w))$ increases gradually. 
Sulak, H. (2016). An EOQ model with defective items and shortages in fuzzy sets environment. International Journal of Social Sciences and Education Research, 2 (3), 915-929.

Table 1. Variation of s effects on optimal solutions.

\begin{tabular}{cccc}
\hline$s$ & $w^{*}$ & $y^{*}$ & $D F(\tilde{\mathcal{L}}(y, w))$ \\
\hline 50 & 585.72 & 2149.85 & $215,378.6$ \\
55 & 585.72 & 2149.85 & $225,378.6$ \\
60 & 585.72 & 2149.85 & $235,378.6$ \\
65 & 585.72 & 2149.85 & $245,378.6$ \\
70 & 585.72 & 2149.85 & $255,378.6$ \\
75 & 585.72 & 2149.85 & $265,378.6$
\end{tabular}

Similarly, from Table- 2, when the unit selling price of imperfect-quality items $v$ increases, the fuzzy total profit $D F(\tilde{\mathcal{L}}(y, w))$ increases slightly.

Table 2. Variation of $\mathbf{v}$ effects on optimal solutions.

\begin{tabular}{rrrc}
\hline$v$ & $w^{*}$ & $y^{*}$ & $D F(\tilde{\mathcal{L}}(y, w))$ \\
\hline 15 & 585.72 & 2149.85 & $362,294.7$ \\
18 & 585.72 & 2149.85 & $364,219.8$ \\
20 & 585.72 & 2149.85 & $365,503.3$ \\
25 & 585.72 & 2149.85 & $368,711.9$ \\
26 & 585.72 & 2149.85 & $369,353.6$ \\
27 & 585.72 & 2149.85 & $369,995.3$
\end{tabular}

Table 3 shows that when the screening rate $x$ increases, the optimal order size $y^{*}$ and the backorder quantity increases remarkably, but the fuzzy total profit $D F(\tilde{\mathcal{L}}(y, w))$ decreases gradually.

Table 3. Variation of $\mathrm{x}$ effects on optimal solutions.

\begin{tabular}{cccc}
\hline$x$ & $w^{*}$ & $y^{*}$ & $D F(\tilde{\mathcal{L}}(y, w))$ \\
\hline 40000 & 447.40 & 2052.25 & $368,418.4$ \\
50000 & 528.99 & 2109.33 & $368,593.4$ \\
55000 & 559.68 & 2131.17 & $368,657.8$ \\
60000 & 585.72 & 2149.85 & $368,711.9$ \\
65000 & 608.10 & 2166.02 & $368,758.0$ \\
75000 & 644.60 & 2192.61 & $368,832.2$ \\
\hline
\end{tabular}

Table 4 points out that when the percentage of scrap items increases, the fuzzy total profit $D F(\tilde{\mathcal{L}}(y, w))$ decreases slightly, but the optimal order size and the optimal backorder quantity do not change.

Table 4. Variation of $\theta$ effects on optimal solutions.

\begin{tabular}{cccc}
\hline$\theta$ & $w^{*}$ & $y^{*}$ & $D F(\tilde{\mathcal{L}}(y, w))$ \\
\hline .14 & 585.72 & 2149.85 & $369,814.0$ \\
.15 & 585.72 & 2149.85 & $369,630.3$ \\
.18 & 585.72 & 2149.85 & $369,079.3$ \\
.20 & 585.72 & 2149.85 & $368,711.9$ \\
.22 & 585.72 & 2149.85 & $368,344.5$ \\
.24 & 585.72 & 2149.85 & $367,977.1$ \\
\hline
\end{tabular}

From the results we can also see that when the triangular fuzzy numbers are used instead of trapezoidal fuzzy numbers, $D F(\tilde{\mathcal{L}}(y, w))$ is more sensitive. 
Sulak, H. (2016). An EOQ model with defective items and shortages in fuzzy sets environment. International Journal of Social Sciences and Education Research, 2 (3), 915-929.

If we plot the fuzzy total profit per unit time function $D F \tilde{\mathcal{L}}(y, w)$ with some values of $y$ and $w$ i.e. $y=1600$ to 2500 with equal interval $w=450$ to 600 , then we have strictly concave graph of total profit function $D F \tilde{\mathcal{L}}(y, w)$ given below.

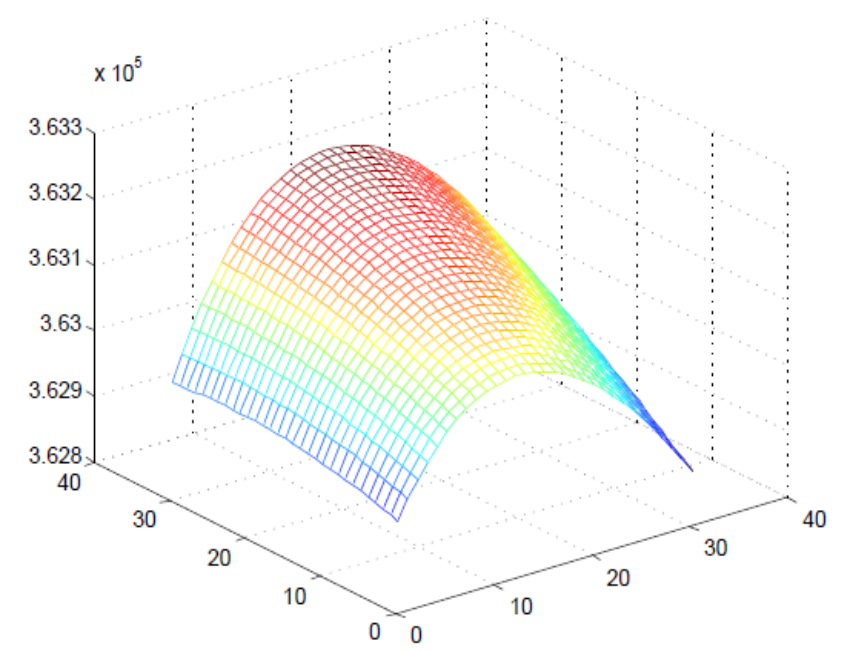

Figure 1. Plot of Fuzzy Total Profit $D F \widetilde{\mathcal{L}}(y, w)$ vs. y and w

\section{Summary and conclusions}

In most of the inventory models, system parameters are described as crisp values or having crisp statistical distributions to reflect uncertainty. Because of resolving the uncertainty which has a significant importance for the decision makers, several researhes have proposed different inventory problems in fuzzy sets environment. In this paper, a fuzzy model for an inventory problem with defective items and shortages is developed. In the model, the demand, defective rate, ordering cost, unit cost, disposal cost, holding cost, backordering cost and screening cost are considered as triangular and trapezoidal fuzzy numbers. The fuzzy model are defuzzified by the GMIR method to find the estimate of the total profit per unit in the fuzzy sense. Numerical examples are provided for developed model, and effects of changes of selling price of good and imperfect quality items, percentage of scrap items and screening rate in optimal policy are studied.

From the results of numerical examples, the percentage of scrap items and screening rate should be reduced as much as possible by decision makers to increase the total profit. The results of the examples applied this model also show that when the triangular fuzzy numbers are used instead of trapezoidal fuzzy numbers, the sensitiveness in the total profit and the values of optimal order size and optimal backorder quantity is comparatively less due to the fuzziness in the components.

\section{Appendix A}

Let consider the following Hessian matrix $(H)$ :

$$
H=\left[\begin{array}{ll}
\frac{\partial^{2} \mathcal{L}(y, w)}{\partial y^{2}} & \frac{\partial^{2} \mathcal{L}(y, w)}{\partial y \partial w} \\
\frac{\partial^{2} \mathcal{L}(y, w)}{\partial w \partial y} & \frac{\partial^{2} \mathcal{L}(y, w)}{\partial w^{2}}
\end{array}\right] .
$$


Sulak, H. (2016). An EOQ model with defective items and shortages in fuzzy sets environment. International Journal of Social Sciences and Education Research, 2 (3), 915-929.

If $[y w] \times[H] \times\left[\begin{array}{c}y \\ w\end{array}\right]<0$, for any $y, w \neq 0$, then the function of $\mathcal{L}(y, w)$ is strictly concave. The elements of Hessian matrix are

$$
\frac{\partial^{2} \mathcal{L}(y, w)}{\partial y^{2}}=-\frac{2 D k(1 /(1-p))+(h+\pi)(1 /(1-p-D / x)) w^{2}}{y^{3}},
$$

In here, since the partial derivatives of $\frac{\partial \mathcal{L}(y, w)}{\partial y}$ and $\frac{\partial \mathcal{L}(y, w)}{\partial w}$ are continuous for all $w$ and $y>0$, and the second order partial derivatives $\frac{\partial^{2} \mathcal{L}(y, w)}{\partial y \partial w}$ and $\frac{\partial^{2} \mathcal{L}(y, w)}{\partial w \partial y}$ are continuous for all $w$ and $y>0$, then we have

$$
\begin{aligned}
& \frac{\partial^{2} \mathcal{L}(y, w)}{\partial y \partial w}=\frac{\partial^{2} \mathcal{L}(y, w)}{\partial w \partial y}=\frac{(h+\pi)(1 /(1-p-D / x)) w}{y^{2}}, \\
& \frac{\partial^{2} \mathcal{L}(y, w)}{\partial w^{2}}=-\frac{(h+\pi)(1 /(1-p-D / x))}{y}
\end{aligned}
$$

and

$$
[y w] \times[H] \times\left[\begin{array}{l}
y \\
w
\end{array}\right]=-\frac{2(1 /(1-p)) k D}{y}<0 .
$$

Therefore, the function of $\mathcal{L}(y, w)$ is strictly concave, thus, $y^{*}$ and $w^{*}$ which make $\mathcal{L}(y, w)$ maksimum have single values.

\section{Appendix B}

Let consider the following Hessian matrix $(H)$ :

$$
H=\left[\begin{array}{ll}
\frac{\partial^{2} D F(\tilde{\mathcal{L}}(y, w))}{\partial y^{2}} & \frac{\partial^{2} D F(\tilde{\mathcal{L}}(y, w))}{\partial y \partial w} \\
\frac{\partial^{2} D F(\tilde{\mathcal{L}}(y, w))}{\partial w \partial y} & \frac{\partial^{2} D F(\tilde{\mathcal{L}}(y, w))}{\partial w^{2}}
\end{array}\right] .
$$

If $[y w] \times[H] \times\left[\begin{array}{l}y \\ w\end{array}\right]<0$, for any $y, w \neq 0$, then the function of $D F(\tilde{\mathcal{L}}(y, w))$ is strictly concave. The elements of Hessian matrix are

$$
\begin{gathered}
\frac{\partial^{2} D F(\tilde{\mathcal{L}}(y, w))}{\partial y^{2}}=-\left\{\left(\frac{\left(h_{1}+\pi_{1}\right)}{\left(q_{4}-\frac{D_{1}}{x}\right)}+\frac{2\left(h_{2}+\pi_{2}\right)}{\left(q_{3}-\frac{D_{2}}{x}\right)}+\frac{2\left(h_{3}+\pi_{3}\right)}{\left(q_{2}-\frac{D_{3}}{x}\right)}+\frac{\left(h_{4}+\pi_{4}\right)}{\left(q_{1}-\frac{D_{4}}{x}\right)}\right) \frac{w^{2}}{2}\right. \\
\left.+\frac{D_{1} K_{1}}{q_{4}}+\frac{2 D_{2} K_{2}}{q_{3}}+\frac{2 D_{3} K_{3}}{q_{2}}+\frac{D_{4} K_{4}}{q_{1}}\right\} \frac{1}{3 y^{3}},
\end{gathered}
$$

Similarly, since the partial derivatives of $\frac{\partial D F(\tilde{\mathcal{L}}(y, w))}{\partial y}$ and $\frac{\partial D F(\tilde{\mathcal{L}}(y, w))}{\partial w}$ are continuous for all $w$ and $y>0$, and the second order partial derivatives $\frac{\partial^{2} D F(\tilde{\mathcal{L}}(y, w))}{\partial y \partial w}$ and $\frac{\partial^{2} D F(\tilde{\mathcal{L}}(y, w))}{\partial w \partial y}$ are continuous for all $w$ and $y>0$, then we have

$$
\begin{aligned}
& \frac{\partial^{2} D F(\tilde{\mathcal{L}}(y, w))}{\partial y \partial w}=\frac{\partial^{2} D F(\tilde{\mathcal{L}}(y, w))}{\partial w \partial y}=\left(\frac{\left(h_{1}+\pi_{1}\right)}{\left(q_{4}-\frac{D_{1}}{x}\right)}+\frac{2\left(h_{2}+\pi_{2}\right)}{\left(q_{3}-\frac{D_{2}}{x}\right)}+\frac{2\left(h_{3}+\pi_{3}\right)}{\left(q_{2}-\frac{D_{3}}{x}\right)}+\frac{\left(h_{4}+\pi_{4}\right)}{\left(q_{1}-\frac{D_{4}}{x}\right)}\right) \frac{w}{6 y^{2}} . \\
& \frac{\partial^{2} D F(\tilde{\mathcal{L}}(y, w))}{\partial w^{2}}=-\left(\frac{\left(h_{1}+\pi_{1}\right)}{\left(q_{4}-\frac{D_{1}}{x}\right)}+\frac{2\left(h_{2}+\pi_{2}\right)}{\left(q_{3}-\frac{D_{2}}{x}\right)}+\frac{2\left(h_{3}+\pi_{3}\right)}{\left(q_{2}-\frac{D_{3}}{x}\right)}+\frac{\left(h_{4}+\pi_{4}\right)}{\left(q_{1}-\frac{D_{4}}{x}\right)}\right) \frac{1}{6 y},
\end{aligned}
$$

and 
Sulak, H. (2016). An EOQ model with defective items and shortages in fuzzy sets environment. International Journal of Social Sciences and Education Research, 2 (3), 915-929.

$[y w] \times[H] \times\left[\begin{array}{l}y \\ w\end{array}\right]=-\left(\frac{D_{1} K_{1}}{q_{4}}+\frac{2 D_{2} K_{2}}{q_{3}}+\frac{2 D_{3} K_{3}}{q_{2}}+\frac{D_{4} K_{4}}{q_{1}}\right) \frac{1}{3 y}<0$.

Since the function of $D F(\tilde{\mathcal{L}}(y, w))$ is strictly concave, this function attains the maximum values only at the point $\left(y^{*}, w^{*}\right)$.

\section{References}

Björk KM. (2008). The economic production quantity problem with a finite production rate and fuzzy cycle time. In Proceedings of the 41st annual Hawaii International Conference on System Sciences, 68-77.

Björk KM. (2009). An analytical solution to a fuzzy economic order quantity problem. International Journal of Approximate Reasoning, 50, 485-493.

Chan WM, Ibrahim RN, Lochert PB. (2003). A new EPQ model: integrating lower pricing, rework and reject situations. Production Planning and Control, 14(7), 588-595.

Chang HC. (2004). An application of fuzzy sets theory to the EOQ model with imperfect quality items. Computers and Operations Research, 31, 2079-2092.

Chang SC, Yao JS, Lee HM. (1998). Economic reorder point for fuzzy backorder quantity. European Journal of Operational Research, 109, 183-202.

Chen HC, Wang CC, Chang SM. (2007). Fuzzy economic production quantity model for items with imperfect quality. International Journal of Innovative Computing, Information and Control, 3(1), 85-95.

Chen S H, Chang SM. (2008). Optimization of fuzzy production inventory model with unrepairable defective products. International Journal of Production Economics, 113(2), 887-894.

Chen SH, Hesieh CH. (1998). Graded mean integration representation of generalized fuzzy numbers. In: Proceedings of the Sixth Conference on Fuzzy Theory and Its Applications, Chinese Fuzzy Systems Association, 1-6.

Chen SH, Wang CC, Arthur R. (1996) Backorder fuzzy inventory model under functional principle. Information Sciences, 95, 71-79.

Chen SH, Wang ST, Chang SM. (2005). Optimization of fuzzy production inventory model with repairable defective products under crisp or fuzzy production quantity. International Journal of Operations Research, 2, 31-37.

Chen SH. (1985). Operations on fuzzy numbers with function principle. Tamkang Journal of Management Sciences, 6(1), 13-25.

Chiu YP. (2003). Determining the optimal lot size for the finite production model with random defective rate, the rework process and backlogging. Engineering Optimization, 35(4), 427-437.

Chung KJ, Hou KL. (2003). An optimal production run time with imperfect production process and allowable shortages. Computers and Operations Research, 30, 483-490.

Eroglu A, Ozdemir G. (2007). An economic order quantity model with defective items and shortages. International Journal of Production Economics, 106, 544-549.

Eroglu A, Sutcu A, Sulak H. (2008). An economic production quantity model with random defective rate in imperfect production processes. Journal of Faculty of Engineering and Architecture, 23(4), 923-929. (in Turkish)

Goyal SK, Cardenas-Barron LE. (2002). Note on economic production quantity model for items with imperfect quality-a practical approach. International Journal of Production Economics, 77, 85-87.

Halim KA, Giri BC, Chaudhuri KS. (2009). Fuzzy EPQ models for an imperfect production system. International Journal of Systems Science, 40, 45-52.

Hayek PA, Salameh MK. (2001). Production lot sizing with the reworking of imperfect quality items produced. Production Planning and Control, 12(6), 584-590.

Hesieh CH. (2002). Optimization of fuzzy production inventory models. Information Sciences, 146, 29-40. 
Sulak, H. (2016). An EOQ model with defective items and shortages in fuzzy sets environment. International Journal of Social Sciences and Education Research, 2 (3), 915-929.

Hu J, Guo C, Xu R, Ji Y. (2010). Fuzzy economic order quantity model with imperfect quality and service level. Chinese Control and Decision Conference, 4042-4047.

Jaber MY, Zanoni S, Zavanella LE. (2014). Economic order quantity model for imperfect items with buy and repair options. International Journal of Production Economics, 155, 126-131.

Jaggi CK, Pareek S, Sharma A, Nidhi. (2012). Fuzzy inventory model for deteriorating items with timevarying demand and shortages. American Journal of Operational Research, 2(6), 81-92.

Kazemi N, Ehsani E, Jaber MY. (2010). An inventory model with backorders with fuzzy parameters and decision variables. International Journal of Approximate Reasoning, 51, 964-972.

Khan M, Jaber MY, Guiffrida AL, Zolfaghari S. (2011). A review of the extensions of a modified EOQ model for imperfect quality items. International Journal of Production Economics, 132, 1-12.

Kim CH, Hong Y. (1999). An optimal production run lenght in deteriorating production processes. International Journal of Production Economics, 58, 183-189.

Kumar RS, Goswami A. (2013) Fuzzy stochastic EOQ inventory model for items with imperfect quality and shortages are backlogged. Advanced Modeling and Optimization, 15(2), 261-279.

Lin DC, Yao JS. (2000). Fuzzy economic production for production inventory. Fuzzy Sets and Systems, $111,465-495$.

Mahata GC, Goswami A. (2013). Fuzzy inventory models for items with imperfect quality and shortage backordering under crisp and fuzzy decision variables. Computers and Industrial Engineering, 64, 190199.

Mizumoto M, Tanaka K. (1979). Some properties of fuzzy numbers. Advances in Fuzzy Set Theory and Applications, 153-164.

Papachristos S, Konstantaras I. (2006). Economic ordering quantity models for items with imperfect quality. International Journal of Production Economics, 100(1), 148-154.

Park K. S. (1987). Fuzzy-set theoretic interpretation of economic order quantity. IEEE Transactions on Systems, Man and Cybernetics, 17, 1082-1084.

Porteus EL. (1986). Optimal lot sizing, process quality improvement and setup cost reduction. Operations Research, 34, 137-144.

Rosenblatt MJ, Lee HL. (1986). Economic production cycles with imperfect production processes. IEE Transactions, 18, 48-55.

Salameh MK, Jaber MY. (2000) Economic production quantity model for items with imperfect quality. International Journal of Production Economics, 64, 59-64.

Sulak, H, Eroğlu, A, Bayhan, M, Avcı, MA. (2015). An Economic Order Quantity Model for Defective Items under Permissible Delay in Payments and Shortage. International Journal of Academic Research in Business and Social Sciences, 5(1), 302-316.

Vijayan T, Kumaran M. (2009). Fuzzy economic order time models with random demand. International Journal of Approximate Reasoning, 50, 529-540.

Vijayan, T., Kumaran, M. (2008). Inventory models with a mixture of backorders and lost sales under fuzzy cost. European Journal of Operational Research, 189, 105-119.

Yao JS, Chiang J. (2003). Inventory without backorder with fuzzy total cost and fuzzy storing cost defuzzified by centroid and signed distance. European Journal of Operations Research, 148, 401-409.

Zadeh L. (1965). Fuzzy sets. Information and Control, 8(3), 338-353. 